\title{
Comment on "Superconductivity at low density near a ferroelectric quantum critical point: Doped $\mathrm{SrTiO}_{3} "$
}

\author{
Jonathan Ruhman ${ }^{1}$ and Patrick A. Lee ${ }^{2}$ \\ 1. Department of Physics, Bar Ilan University, Ramat Gan 5290002, Israel \\ 2. Department of Physics, Massachusetts Institute of Technology, Cambridge, MA 02139 USA
}

\begin{abstract}
Wölfle and Balatsky Phys. Rev. B 98, 104505 (2018) have proposed a microscopic pairing mechanism for doped $\mathrm{SrTiO}_{3}$ (STO) based on the gradient coupling of electronic density to the soft TO phonon mode. Since this coupling to TO phonons is usually weak, this conclusion is surprising, especially for a low density superconductor such as STO, where the density of states is small. A crucial step in the argument made by Wölfle and Balatsky is that the displacement vector of the TO mode is not strictly perpendicular to the momentum vector, making a deformation coupling possible. We show that they have made a mistake in computing the eigenvector and have grossly overestimated this lack of orthogonality. When corrected, the coupling is negligible. We also use transport data to put upper bounds on the coupling constant which are much smaller than the estimate by Wölfle and Balatsky. Finally, we also object to their use of the Eliashberg equation when the phonon frequency is larger than the Fermi energy.
\end{abstract}

The microscopic mechanism for superconductivity in doped $\mathrm{SrTiO}_{3}$ (STO) has been recently discussed in Ref. $1 .^{2}$ Central to their discussion is the claim that exchanging the soft transverse optical (TO) mode, that is related to the proximity to the ferroelectric transition, gives a sufficiently large coupling to explain the experimentally observed $T_{c}$, even at very low densities. This is contrary to the statement we made in an earlier publication $^{3}$ that due to the low density of states, the dimensionless electron phonon coupling $\lambda$ via the exchange of TO phonons is too weak to give any reasonable $T_{c}$. In addition, we also stated that while the mechanism of exchanging polar phonon proposed by Gurevich et. al. ${ }^{4}$ may work at intermediate doping densities, even that fails for very low densities when the requirement for adiabaticity of the phonons is respected. The work of Ref. 1 motivated us to study in detail the coupling of TO phonons to electrons near the zone center. We clarified the roles of Coulomb interaction with the ions versus that of a short range pseudo-potential. In the process we found an error made in Ref. 1, which led them to overestimate the coupling strength and we re-affirm our previous conclusion.

In the case of a ferroelectric transition the order parameter is a lattice distortion, i.e. an optical phonon mode. There are three relevant phonon polarizations, which are traditionally labeled as one longitudinal optical (LO) and two transverse optical (TO) modes. The long-ranged dipolar interactions in the LO mode, however, make it stiff and prevent it from becoming soft at the transition ${ }^{5-8}$. Thus, the two TO modes are the dynamical soft boson modes associated with this transition.

To get pairing directly from these soft modes the authors of Ref. 1 used a phenomenological model involving a displacement vector $\boldsymbol{u}_{\boldsymbol{q}}$ and invoked a gradient coupling of the form

$$
H_{e l-p h}=i V_{0} \sum_{\boldsymbol{q}} \boldsymbol{q} \cdot \boldsymbol{u}_{\boldsymbol{q}} \rho_{-\boldsymbol{q}}
$$

where $\boldsymbol{u}$ is the soft phonon displacement and $\rho$ is the electronic density. Clearly, if the polarizations of the TO modes are truly transverse this coupling vanishes. They argued that cubic crystal anisotropy tilts the polarization of the soft modes such that they are never really transverse to $\hat{\boldsymbol{q}}$ except for high symmetry lines. The square of the overlap between the mode polarization $\hat{\boldsymbol{e}}_{T}(\boldsymbol{q})$ and the momentum direction

$$
s \equiv\left\langle\left(\hat{\boldsymbol{e}}_{T}(\boldsymbol{q}) \cdot \hat{\boldsymbol{q}}\right)^{2}\right\rangle
$$

was estimated and found to be of order 0.1. This parameter multiplies the effective BCS coupling in the s-wave channel, which arises from Eq. (1). Thus, their theory depends crucially on the fact that $s$ is order 0.1 and not negligibly small.

It is important to note that for STO the carrier density and therefore the Fermi momentum is very small and the small $q$ limit of Eq. (1) is relevant. Physically we expect the crystalline symmetry to become irrelevant in the $q \rightarrow 0$ limit and the polarization should be strictly perpendicular and parallel to $\boldsymbol{q}$ for the TO and LO mode, respectively. As a result $s$ should approach zero. This is because any deviation from strictly transverse displacement will mix in long range polarization and raise the energy of the TO mode. In the following we prove this analytically and obtain an expression showing that $s$ is proportional to $q^{4}$ (see Eq. (14) below). Setting $q$ to be the $k_{F}$ for a given density, we find that for a density $n=10^{20} \mathrm{~cm}^{-3}, s$ is smaller than $10^{-5}$ and drops drastically with density. Thus, the authors of Ref. 1 have miscalculated $s$ by orders of magnitude. Their estimate of $\lambda$ is off by the same amount and their conclusion that $T_{c}$ in STO can be explained by coupling to the soft TO phonon should be reevaluated.

In what follows, we also pin-point the error made in Ref. 1. In evaluating the TO mode eigenvectors, they did not take into account the long-ranged Coulomb forces, which are dominant in the limit $q \rightarrow 0$. Consequently, they find $s$ to be independent on $q$ and substantial, as in non-polar materials.

We then go beyond the phenomenological model and 
consider the full problem of a crystal structure with multiple ions, as is appropriate for STO. We find that the results of the phenomenological model applies to the Coulomb potential considered by Wölfle and Balatsky, which gives a negligible contribution to the coupling. There exists a second kind of contribution from the short range pseudo-potential, which is not generally forced to vanish in the same way. However, for cubic and tetragonal crystals it takes the form Eq. (1) and is also negligible due to the same factor.

Another issue in Ref. 1 regards the other pairing mechanism they have considered: the high frequency LO mode. The authors continued to use the Eliashberg equations even when the phonon frequency exceeds the Fermi energy by one and two orders of magnitude. We argue at the end of this comment that in the absence of proof to the contrary, conventional wisdom should apply and the Eliashberg equation should be restricted to the adiabatic limit.

\section{OPTICAL-PHONON DISPERSION CLOSE TO THE ZONE CENTER IN A POLAR CUBIC CRYSTAL}

In this section we present the action for optical phonons in $\mathrm{SrTiO}_{3}$ from which the dispersion and polarization vectors near the zone center can be derived. Because the error in Ref. 1 arises from ambiguity in the inclusion of long-ranged forces in the action formalism we first include dispersion terms based on short-ranged forces, which are identical to the ones in Ref. 1. Then, in the next step we show how to include the long-ranged forces.

We start from the action

$$
\mathcal{S}_{u}=\frac{1}{2} \sum_{\omega, \boldsymbol{q}} u_{-\boldsymbol{q}}^{j}\left[\omega^{2} \delta_{j l}-A_{j l}(\boldsymbol{q})\right] u_{\boldsymbol{q}}^{l}
$$

Here $\boldsymbol{u}$ is the optical displacement which is proportional to the dipolar polarization and $A_{i j}(\boldsymbol{q})$ is the dispersion matrix given by

$$
A_{i j}(\boldsymbol{q})=\omega_{T}^{2} \delta_{i j}+c_{T}^{2}\left(q^{2} \delta_{i j}-q_{i} q_{j}\right)+c_{L}^{2} q_{i} q_{j}+\alpha q_{i}^{2} \delta_{i j}
$$

$c_{L}$ and $c_{T}$ are the longitudinal and transverse phonon velocities, respectively, $\omega_{T}$ is the optical gap at the zone center and $\alpha$ arises from the cubic crystal fields, which breaks rotational symmetry. The model above is identical to the one used by Wölfle and Balatsky ${ }^{1}$ (see Eq. A15). The two are related using the identities $\boldsymbol{P}=\omega_{D} \boldsymbol{u}, \omega_{D}=33 \mathrm{meV}, \lambda_{1}=\left(c_{L}^{2}+\alpha\right) / \omega_{D}^{2}=8 \AA^{2}$, $\lambda_{2}=\left(c_{L}^{2}-c_{T}^{2}\right) / \omega_{D}^{2}=1 \AA^{2}, \lambda_{3}=c_{T}^{2} / \omega_{D}^{2} 2 \AA^{2}$ and $\tau=\omega_{T}^{2} / \omega_{D}^{2}$. In the first identity $\boldsymbol{P}$ is essentially the dipolar polarization associated with the optical distortion field $\boldsymbol{u}$. Note that the numbers quoted here are copied from Ref. 1, and we have not independently verified their accuracy.
We also note that we insist on modifying the notations of Ref. 1 to the ones in Eq. (3) because in this representation the role of the crystal anisotropy $\alpha$ becomes clear. This also allows us to seperate the terms which are fully rotationally symmetric form this term, which will become useful in the next section.

To add the effects of the long-ranged dipolar forces associated with the optical distortion we write their coupling to the electric field

$$
\mathcal{S}_{E-u}=-\sum_{\boldsymbol{q}, \omega} \boldsymbol{P}_{\boldsymbol{q}} \cdot \boldsymbol{E}_{-\boldsymbol{q}}=-\omega_{D} \sum_{\boldsymbol{q}, \omega} \boldsymbol{u}_{\boldsymbol{q}} \cdot \boldsymbol{E}_{-\boldsymbol{q}}
$$

It is crucial to note that we assume that $\boldsymbol{E}$ is static (we neglect dynamics of the electromagnetic fields). Thus, $\boldsymbol{E}$ stems from a potential $\varphi$, such that $\boldsymbol{E}_{\boldsymbol{q}}=i \boldsymbol{q} \cdot \varphi_{\boldsymbol{q}}$. Consequently, $\boldsymbol{E}_{\boldsymbol{q}}$ is strictly a longitudinal vector. When taking the dot-product with $\boldsymbol{u}_{\boldsymbol{q}}$ in the coupling term (5), it nullifies the components perpendicular to $\boldsymbol{q}$ and we can equivalently write the coupling term as

$$
\mathcal{S}_{E-u}=-\omega_{D} \sum_{\boldsymbol{q}, \omega} \hat{\boldsymbol{q}} \cdot \boldsymbol{u}_{\boldsymbol{q}} E_{-\boldsymbol{q}}
$$

To obtain the effective dispersion we would like to integrate out the electric field. To this end, we recall the action of the of the electric field, which is the energy density (again, neglecting dynamics)

$$
\mathcal{S}_{E}=\frac{\varepsilon_{\infty}}{8 \pi} \sum_{\boldsymbol{q}, \omega}\left|E_{\boldsymbol{q}}\right|^{2}
$$

Completing a square between Eq. (7) and Eq. (6) and integrating over the electric field we obtain a shift in the field $\boldsymbol{u}$

$$
\delta \mathcal{S}_{u}=-\frac{1}{2} \sum_{\omega \boldsymbol{q}} \frac{4 \pi \omega_{D}^{2}}{\varepsilon_{\infty}} \hat{q}_{j} \hat{q}_{l} u_{\boldsymbol{q}}^{j} u_{-\boldsymbol{q}}^{l}
$$

Thus, the combined effect of long and short ranged physics leads to the action

$$
\tilde{\mathcal{S}}_{u}=\mathcal{S}_{u}+\delta \mathcal{S}_{u}=\frac{1}{2} \sum_{\omega, \boldsymbol{q}} u_{-\boldsymbol{q}}^{j}\left[\omega^{2} \delta_{j l}-\tilde{A}_{j l}(\boldsymbol{q})\right] u_{\boldsymbol{q}}^{l}
$$

where the corrected dispersion matrix is given by ${ }^{5}$

$$
\begin{aligned}
\tilde{A}_{i j}(\boldsymbol{q})=\omega_{T}^{2} \delta_{i j} & +c_{T}^{2}\left(q^{2} \delta_{i j}-q_{i} q_{j}\right) \\
& +\left(\omega_{L}^{2}-\omega_{T}^{2}\right) \hat{q}_{i} \hat{q}_{j}+c_{L}^{2} q_{i} q_{j}+\alpha q_{i}^{2} \delta_{i j}
\end{aligned}
$$

The LO and TO modes are the eigenstates of this equation. Note that as expected, the LO frequecy has been sifted from $\omega_{T}$ to $\omega_{L} \equiv \sqrt{\omega_{T}^{2}+4 \pi \omega_{D}^{2} / \varepsilon_{\infty}}$.

In $\mathrm{SrTiO}_{3}$ this mass term is quite large. Neglecting complications coming from multiple modes, we can assign $\omega_{L} \approx 100 \mathrm{meV}$, while $\omega_{T} \approx 2 \mathrm{meV}$. The quantum critical point is obtained by taking $\omega_{T} \rightarrow 0$. The dynamics of the longitudinal component, however, are described by a finite frequency mode. 


\section{ESTIMATION OF THE POLARIZATION VECTORS AND DISPERSION}

The polarization of the optical phonon branches are dictated by the dispersion matrix $\tilde{A}(\boldsymbol{q})$. The main mistake of the authors of Ref. 1 is that they computed the soft mode polarization vectors $\hat{e}_{T}$ using the matrix $A(\boldsymbol{q})$ in action (3) and not $\tilde{A}(\boldsymbol{q})$ in the action Eq. (9) (see Eq.(A30) in their appendix). As a result they obtain that the polarization vectors are independent on the magnitude of the momentum $q$. However, upon inspection of Eq.(9) we find that in the limit of $q \rightarrow 0$ the mass term Eq. (8) remains finite, unlike the anisotropy term $\alpha$. In this limit the gapped mode is purely longitudinal and decoupled from the soft transverse modes, thus nullifying the coupling shown in Eq. (1) to the TO modes. This conclusion holds for any TO mode that involves a dipolar excitation, whether it is soft or not.

To see this let us estimate the polarization vectors close to $q=0$. We first separate the dispersion matrix into a fully rotational symmetric part and the anisotropy term

$$
\tilde{A}(\boldsymbol{q})=M(\boldsymbol{q})+\delta M(\boldsymbol{q})
$$

Here $M_{i j}(\boldsymbol{q})=\omega_{T}^{2} \delta_{i j}+c_{T}^{2}\left(q^{2} \delta_{i j}-q_{i} q_{j}\right)+\left(\omega_{L}^{2}-\omega_{T}^{2}\right) \frac{q_{i} q_{j}}{q^{2}}+$ $c_{L}^{2} q_{i} q_{j}$ is the isotropic matrix and $\delta M_{i j}(\boldsymbol{q})=\alpha q_{i}^{2} \delta_{i j}$ is responsible for breaking rotational symmetry and giving a finite value to Eq. (2). The eigenvalues and eigenvectors of the matrix $M(\boldsymbol{q})$ are given by

$$
\begin{aligned}
& \hat{\boldsymbol{n}}_{L}=\frac{1}{q}\left(q_{x}, q_{y}, q_{z}\right) \quad ; \quad \lambda_{L}(q)=\omega_{L}^{2}+c_{L}^{2} q^{2} \\
& \hat{\boldsymbol{n}}_{T 1}=\frac{\left(q_{y}-q_{z}, q_{z}-q_{x}, q_{x}-q_{y}\right)}{\sqrt{\left(q_{y}-q_{z}\right)^{2}+\left(q_{z}-q_{x}\right)^{2}+\left(q_{x}-q_{y}\right)^{2}}} \quad ; \quad \lambda_{T}(q)=\omega_{T}^{2}+c_{T}^{2} q^{2} \\
& \hat{\boldsymbol{n}}_{T 2}=\hat{\boldsymbol{n}}_{T 1} \times \hat{\boldsymbol{n}}_{L}
\end{aligned}
$$

This is the exact eigensystem for the case of $\delta M=0$ (or equivalently, $\alpha=0$ ). To compute the eigenbasis for $\alpha \neq 0$ we treat $\delta M$ as a perturbation

$$
\begin{aligned}
\delta \hat{\boldsymbol{n}}_{T 1} & =\frac{\hat{\boldsymbol{n}}_{L}^{\mathrm{T}} \cdot \delta M(\boldsymbol{q}) \cdot \hat{\boldsymbol{n}}_{T 1}}{\lambda_{T}(q)-\lambda_{L}(q)} \hat{\boldsymbol{n}}_{L} \\
\delta \hat{\boldsymbol{n}}_{T 2} & =\frac{\hat{\boldsymbol{n}}_{L}^{\mathrm{T}} \cdot \delta M(\boldsymbol{q}) \cdot \hat{\boldsymbol{n}}_{T 2}}{\lambda_{T}(q)-\lambda_{L}(q)} \hat{\boldsymbol{n}}_{L}
\end{aligned}
$$

such that $\hat{\boldsymbol{e}}_{T 1,2}=\hat{\boldsymbol{n}}_{T 1,2}+\delta \hat{\boldsymbol{n}}_{T 1,2}+\mathcal{O}\left(\delta M^{2}\right)$. The perturbative approach can be justified in the limit of $q \rightarrow 0$ by noting that $\lim _{q \rightarrow 0} \delta M(\boldsymbol{q})=0$ in contrast to $M(\boldsymbol{q})$ that remains finite, and thus, the eigenbasis (12) becomes exact in this limit. Thus, the perturbation theory is valid in the limit $q^{2} \ll\left(\lambda_{L}-\lambda_{T}\right) / \alpha \approx \omega_{L}^{2} / \alpha \approx 0.7(2 \pi / a)^{2}$.

A direct computation using Eq. (13) gives

$$
s \equiv \int \frac{d \Omega}{4 \pi}\left(\hat{\boldsymbol{q}} \cdot \hat{\boldsymbol{e}}_{T 1}\right)^{2}=\frac{r \alpha^{2} q^{4}}{4 \pi\left[\lambda_{L}(q)-\lambda_{T}(q)\right]^{2}} \approx \frac{r \alpha^{2} q^{4}}{4 \pi \omega_{L}^{4}}
$$

where $r \approx 0.239 \ldots$ can be expressed as an integral over a lengthy expression of trigonometric functions. Note that in the last line we assumed $\omega_{L}^{2} \gg c_{T}^{2} q^{2}, c_{L}^{2} q^{2}, \omega_{T}^{2}$.

Now lets make some estimates. In $\mathrm{SrTiO}_{3}$ the longitudinal phonon frequency should be $\omega_{L}=100 \mathrm{meV}$. We can overestimate the parameter $\alpha$ by taking it to be $\alpha=c_{T}^{2}$, where the velocity of the transverse mode is $c_{T} \sim 5 \mathrm{meV} \mathrm{nm}$ (this implies that the dispersion is very anisotropic). Finally, to get a number we estimate this average at $q=2 k_{F}$, where $k_{F}=\left(3 \pi^{2} n\right)^{1 / 3}$. At a density $n=10^{20} \mathrm{~cm}^{-3}$ we find that $\frac{\alpha q^{2}}{\omega_{L}^{2}} \sim 0.01$, and therefore the overlap squared averages to $2 \times 10^{-6}$. For $n=10^{17} \mathrm{~cm}^{-3}$, we get $\frac{\alpha q^{2}}{\omega_{L}^{2}} \sim 0.0001$ and therefore the overlap squared is $2 \times 10^{-10}$. In contrast Wölfle and Balatsky estimated $s \approx 0.1$. Clearly, if they take into account this correction the pairing they found in the s-wave channel will become negligibly small.

We emphasize that the $q^{4}$ dependence in Eq. (14) holds only for modes that create a dipolar excitation within the unit cell. It is this dipolar coupling that forces the displacement vector to be nearly perpendicular to $\boldsymbol{q}$. As an example, consider the $6 \mathrm{meV}$ TO mode that is associated with the cubic to tetragonal transition at $100 \mathrm{~K}$. This mode originate as a zone corner mode in the cubic phase that is folded to the zone center and produces a quadrupole moment rather than dipole in the unit cell. In this case the factor $s$ can be finite in the limit of small $q$. A reasonably large $\lambda$ can be obtained by exchanging this mode at intermediate and high density ${ }^{9}$ and may supplement the polar phonon mechanism ${ }^{4}$ and contribute to $T_{c}$, even though $\lambda$ will still be small at low density due to the small density of states. Indeed, there is evidence that this mode contributes to the transport scattering rate around $50 \mathrm{~K} .^{10}$

\section{A GENERAL FORMULATION OF THE DEFORMATION POTENTIAL}

Next we consider the general problem of the coupling to a TO mode in a crystal with multiple ions in the unit 
cell. we first consider the general potential caused by a deformation. We make the rigid ionic potential ap- proximation, i.e., we write the potential induced on the electrons by the deformation of the set of lattice deformations $\left\{\boldsymbol{U}_{j \alpha}\right\}$ as

$$
V_{e l-p h}(\boldsymbol{r})=\sum_{j \alpha}\left[V_{\alpha}\left(\boldsymbol{r}-\boldsymbol{R}_{j \alpha}-\boldsymbol{U}_{j \alpha}\right)-V\left(\boldsymbol{r}-\boldsymbol{R}_{j \alpha}\right)\right] \approx \sum_{j \alpha} \boldsymbol{U}_{j \alpha} \cdot \nabla V_{\alpha}\left(\boldsymbol{r}-\boldsymbol{R}_{j \alpha}\right)
$$

where $\boldsymbol{R}_{j \alpha}=\boldsymbol{R}_{j}+\boldsymbol{\tau}_{\alpha}$ is the position of the $\alpha$ ion at unit cell $j$ and $V_{\alpha}(\boldsymbol{r})$ is the potential it induces. The precise form of this potential will be discussed later in more detail. Thus, the matrix element for transitions between electronic states due to this potential is thus given by

$$
\begin{aligned}
\left\langle\boldsymbol{k} ; n\left|V_{e l-p h}(\boldsymbol{r})\right| \boldsymbol{k}^{\prime} ; n^{\prime}\right\rangle & =\sum_{j} \int d^{3} r \psi_{\boldsymbol{k} n}^{*}(\boldsymbol{r}) \boldsymbol{U}_{j \alpha} \cdot \nabla V_{\alpha}\left(\boldsymbol{r}-\boldsymbol{R}_{j \alpha}\right) \psi_{\boldsymbol{k}^{\prime} n^{\prime}}(\boldsymbol{r}) \\
& =\frac{i}{\Omega^{2}} \sum_{\boldsymbol{q}} \int d^{3} r \boldsymbol{q} \cdot\left(\sum_{j} \boldsymbol{U}_{j \alpha} e^{i \boldsymbol{q} \cdot \boldsymbol{R}_{j \alpha}}\right) V(\boldsymbol{q}) e^{i\left(\boldsymbol{q}+\boldsymbol{k}-\boldsymbol{k}^{\prime}\right) \cdot \boldsymbol{r}} \chi_{\boldsymbol{k} n}(\boldsymbol{r}) \chi_{\boldsymbol{k}^{\prime} n^{\prime}}^{*}(\boldsymbol{r}) \\
& =\frac{i}{\Omega^{3 / 2}} \sum_{l} e^{i\left(\boldsymbol{q}+\boldsymbol{k}-\boldsymbol{k}^{\prime}\right) \boldsymbol{R}_{l}} \sum_{\boldsymbol{q}} \frac{1}{v_{u c}} \int_{u c} d^{3} r \boldsymbol{q} \cdot \boldsymbol{U}_{\boldsymbol{q} \alpha} V_{\alpha}(\boldsymbol{q}) e^{i\left(\boldsymbol{q}+\boldsymbol{k}-\boldsymbol{k}^{\prime}\right) \cdot \boldsymbol{r}} \chi_{\boldsymbol{k} n}(\boldsymbol{r}) \chi_{\boldsymbol{k}^{\prime} n^{\prime}}^{*}(\boldsymbol{r}) \\
& =\frac{i}{\Omega^{3 / 2}} \sum_{\boldsymbol{G}} V_{\alpha}(\boldsymbol{Q}) \boldsymbol{Q} \cdot \boldsymbol{U}_{\boldsymbol{k}-\boldsymbol{k}^{\prime} \alpha} \int_{u c} \frac{d^{3} r}{v_{u c}} e^{i \boldsymbol{G} \cdot \boldsymbol{r}} \chi_{\boldsymbol{k} n}(\boldsymbol{r}) \chi_{\boldsymbol{k}^{\prime} n^{\prime}}^{*}(\boldsymbol{r})
\end{aligned}
$$

Here $\Omega=N a^{3}$ is the total volume, $a$ is the lattice constant, $\boldsymbol{Q}=\boldsymbol{k}^{\prime}-\boldsymbol{k}+\boldsymbol{G}$ represents the conservation of crystal momentum, the $\psi_{\boldsymbol{k} n}(\boldsymbol{r})=\frac{e^{i \boldsymbol{k} \cdot \boldsymbol{r}}}{\sqrt{\Omega}} \chi_{\boldsymbol{k} n}(\boldsymbol{r})$ are the Bloch wave functions of an electron in band $n$ and the integral is over the unit-cell. Note that $\boldsymbol{U}_{\boldsymbol{q}, \alpha}=\boldsymbol{U}_{\boldsymbol{q}+\boldsymbol{G}, \alpha}$. Thus, we can define the transition matrix element

$$
M_{n n^{\prime}}^{\boldsymbol{G \alpha}}\left(\boldsymbol{k}, \boldsymbol{k}^{\prime}\right) \equiv \frac{i V_{\alpha}(\boldsymbol{Q})}{v_{u c}} \int_{u c} d^{3} r e^{i \boldsymbol{G} \cdot \boldsymbol{r}} \chi_{\boldsymbol{k} n}(\boldsymbol{r}) \chi_{\boldsymbol{k}^{\prime} n^{\prime}}^{*}(\boldsymbol{r})
$$

such that the electron-phonon coupling is given by

$$
H_{e l-p h}=\frac{1}{\Omega^{3 / 2}} \sum_{\boldsymbol{G \alpha} \boldsymbol{k} \boldsymbol{k}^{\prime}} M_{n n^{\prime}}^{\boldsymbol{G \alpha}}\left(\boldsymbol{k}, \boldsymbol{k}^{\prime}\right)\left(\boldsymbol{k}^{\prime}-\boldsymbol{k}+\boldsymbol{G}\right) \cdot \boldsymbol{U}_{\boldsymbol{k}-\boldsymbol{k}^{\prime} \alpha} c_{\boldsymbol{k} n}^{\dagger} c_{\boldsymbol{k}^{\prime} n^{\prime}}
$$

This expression is quite general. Notice that there are terms proportional to $\boldsymbol{G}$, which allow a finite coupling in the limit $q=\left|\boldsymbol{k}-\boldsymbol{k}^{\prime}\right| \rightarrow 0$. We comment on these terms and inversion symmetry are present, one can show that the sum of two contributions, $\boldsymbol{G}$ and $-\boldsymbol{G}$, cancel each other if also $n=n^{\prime}$ (i.e. intraband scattering). That is, $M_{n n}^{\boldsymbol{G}}(\boldsymbol{k}, \boldsymbol{k})=M_{n n}^{-\boldsymbol{G}}(\boldsymbol{k}, \boldsymbol{k})$. To see this we note that with TRS we have $\chi_{\boldsymbol{k} n}^{*}(\boldsymbol{r})=\chi_{-\boldsymbol{k} n}(\boldsymbol{r})$ and with inversion we have $\chi_{\boldsymbol{k} n}(\boldsymbol{r})=\chi_{-\boldsymbol{k} n}(-\boldsymbol{r})$. Thus, the product $\chi_{\boldsymbol{k} n}^{*}(\boldsymbol{r}) \chi_{\boldsymbol{k} n}(\boldsymbol{r})$ is an even function of $\boldsymbol{r}$, and thus its Fourier series is an even function of $\boldsymbol{G}$. Thus, the conclusion, is that with these symmetries present the electron coupling to transverse optical phonons at zero momentum transfer must include interband processes. at the end of this section, but since the authors of Ref. 1 did not consider the finite $\boldsymbol{G}$ terms we continue to follow their analysis and consider only normal processes with $\boldsymbol{G}=0$. Additionally, they also only considered a single band approximation, therefore they arrive at a result of the form

$$
H_{e l-p h}=i \sum_{\boldsymbol{q} \alpha} V_{\alpha}(\boldsymbol{q}) \boldsymbol{q} \cdot \boldsymbol{U}_{\boldsymbol{q} \alpha} \rho_{-\boldsymbol{q}}
$$

Let us write $V_{\alpha}(\boldsymbol{q})=Z_{\alpha} V_{C}(\boldsymbol{q})+V_{p s, \alpha}(\boldsymbol{q})$ where

$$
V_{C}(\boldsymbol{q})=\frac{4 \pi e^{2}}{\varepsilon_{\infty} q^{2}}
$$

is the Coulomb interaction, $Z_{\alpha}$ is the charge on ion $\alpha$ such that charge neutrality gives $\sum_{\alpha} Z_{\alpha}=0$ and $V_{p s, \alpha}(\boldsymbol{q})$ is what is left over, which we refer to as the pseudo-potential of ion $\alpha$. Then Eq. (19) becomes

$$
H_{e l-p h}=i \sum_{\boldsymbol{q}} V(\boldsymbol{q}) \boldsymbol{q} \cdot \boldsymbol{u}_{\boldsymbol{q}} \rho_{-\boldsymbol{q}}+i \sum_{\boldsymbol{q} \alpha} V_{p s, \alpha}(\boldsymbol{q}) \boldsymbol{q} \cdot \boldsymbol{U}_{\boldsymbol{q} \alpha} \rho_{-\boldsymbol{q}}
$$

where $\boldsymbol{u}_{\boldsymbol{q}}=\sum_{\alpha} Z_{\alpha} \boldsymbol{U}_{\alpha}(\boldsymbol{q})$ is the displacement, which is

proportional to the unit-cell dipole and corresponds to 
the soft phonon discussed in Section II. This discussion there applies: in particular, the average of $\left(\hat{\boldsymbol{q}} \cdot \hat{\boldsymbol{u}}_{\boldsymbol{q}}\right)^{2}$ is proportional to $q^{4}$ as given by Eq. (14). As a result, the contribution from the Coulomb interaction, the only one kept by Wölfle and Balatsky, is entirely negligible. On the other hand, the second term in Eq. (21) is proportional to $\boldsymbol{u}^{\prime} \cdot \boldsymbol{q}$ where $\boldsymbol{u}_{\boldsymbol{q}}^{\prime}=\sum_{\alpha} V_{p s, \alpha}(\boldsymbol{q}) \boldsymbol{U}_{\boldsymbol{q} \alpha}$ and where $V_{p s, \alpha}$ is the short-ranged pseudo potential of ion $\alpha . \boldsymbol{u}^{\prime}$ is in general not parallel to $\boldsymbol{u}$ and does not need to be perpendicular to $\boldsymbol{q}$ even in the $\boldsymbol{q} \rightarrow 0$ limit. However, for cubic and tetragonal crystal structure, as in $\mathrm{SrTiO}_{3}$, symmetry constraints all the individual displacements $\boldsymbol{U}_{\boldsymbol{q} \alpha}$ to be colinear near the zone center. This is clearly the case for $\boldsymbol{q}$ along a symmetry direction such as the $x$ axis and it is easy to see that it continues to hold for arbitrary direction because the force matrix is non-singular in the $\boldsymbol{q}$ goes to zero limit. Thus, $\boldsymbol{u}$ is parallel to all $\boldsymbol{U}_{\boldsymbol{q} \alpha}$ and we may conclude that also this term is suppressed by the same factor of $s \propto q^{4}$ and is therefore negligible.

To conclude, we have shown that the gradient coupling of the soft TO mode to electronic density in $\mathrm{SrTiO}_{3}$ is dramatically suppressed near the zone center. This is due to long-ranged Coulomb forces, which bend the polarization to become truly transverse to $\boldsymbol{q}$. The result holds both for the long-ranged Coulomb repulsion and the short range pseudo potential. We find confirmation to our results in a recent $a b$ initio calculation of the electronphonon coupling in $\mathrm{STO}^{11}$, where the coupling to the TO phonon is found to be weak and decreases at small $q$.

Regarding the terms with finite $\boldsymbol{G}$ in Eq. (18) we note that in the limit of zero momentum transfer $\boldsymbol{k}=\boldsymbol{k}^{\prime}$ and when both time-reversal and inversion symmetry are present, one can show that the sum of two contributions, $\boldsymbol{G}$ and $-\boldsymbol{G}$, cancel each other if $n=n^{\prime}$ (i.e. intraband scattering). That is, $M_{n n}^{G}(\boldsymbol{k}, \boldsymbol{k})=M_{n n}^{-\boldsymbol{G}}(\boldsymbol{k}, \boldsymbol{k})$. To see this we note that with time reversal symmetry we have $\chi_{\boldsymbol{k}_{n}}^{*}(\boldsymbol{r})=\chi_{-\boldsymbol{k} n}(\boldsymbol{r})$ and with inversion we have $\chi_{\boldsymbol{k} n}(\boldsymbol{r})=\chi_{-\boldsymbol{k} n}(-\boldsymbol{r})$. Thus, the product $\chi_{\boldsymbol{k} n}^{*}(\boldsymbol{r}) \chi_{\boldsymbol{k} n}(\boldsymbol{r})$ is an even function of $\boldsymbol{r}$, and thus its Fourier series is an even function of $\boldsymbol{G}$. Concerning interband scattering, for states with $\boldsymbol{k}$ near the zone center, they are either even or odd under inversion. interband scattering between even and odd states is allowed for states near the zone center. However, in STO, the states of interest near the Fermi level are d wave which are even. Therefore we conclude that the finite $\boldsymbol{G}$ processes are negligible in STO at low density.

\section{BOUNDING THE COUPLING USING TRANSPORT DATA}

In this section we roughly estimate the coupling to the TO mode based on the resistivity measurements in Ref. 12. If a coupling of the form Eq. (1) exists we anticipate the phonon limited transport lifetime at tempera- tures higher than the mode frequency

$$
\frac{\hbar}{\tau} \approx 2 \pi k_{B} T \lambda
$$

where $\lambda$ is the BCS coupling strength. It is important however to note that the formula above applies to a flat phonon band. For the soft TO mode this applies only when $k_{F}<\omega_{T} / v_{s} \approx 3 \times 10^{6} \mathrm{~cm}^{-1}$, which corresponds to a density of $10^{18} \mathrm{~cm}^{-3}$. At this density Ref. ${ }^{12}$ measure an inverse lifetime $\tau_{t r}^{-1} \approx 0.5 \mathrm{ps}^{-1}$ at a temperature of $T=30$. Estimating the coupling we get that $\lambda \leq \frac{\hbar / \tau}{\pi k_{B} T} \approx$ 0.04 which is in strong disagreement with the results of Ref. 1, which find $\lambda \sim 0.2$. For higher densities, we can add a factor $\left(\omega_{T} / v_{s} k_{F}\right)^{2}$ to the RHS of Eq (26) to keep scattering by only the modes that are almost flat. For a density of $5 \times 10^{18} \mathrm{~cm}^{-3}$, we still get a bound of $\lambda \sim 0.15$, giving a $T_{c}$ of about $10^{-3} \omega_{T O}$ according to BCS theory.

\section{THE INCLUSION OF THE HIGH-FREQUENCY LO MODE IN THE ELIASHBERG FRAMEWORK}

In addition to the soft TO mode, which was discussed in this comment in great detail, the authors of Ref. 1 have also considered the dynamics of the screened Coulomb potential as a pairing mechanism. In particular, they considered the dynamics associated with the high-frequency LO mode. They argue that even when the phonon frequency is greater than the Fermi energy, the Eliashberg still captures the essential physics up to the cut-off $\omega_{c}$, which is determined by the criterion that the quasi-particles scattering rate is small compared to frequency. We disagree with this and point out that the Eliashberg equation is based on the approximation of keeping the ladder diagrams with the leading logarithmic divergence. For frequencies above the Fermi energy, the ladder diagrams no longer carry the logarithm and many other diagrams contribute equally (for example see the diagrams in Fig.2 of Ref.13). Whether the quasiparticles are well defined or not, is not the only issue.

Historically, Takada ${ }^{14}$ treated the dynamically screened interaction in the same way and integrated the Eliashberg equation up to very high frequencies, to obtain a relatively large $T_{c}$, However, this procedure has been criticized by showing that vertex corrections are large ${ }^{13}$. In Ref. 1 , the consequence of taking a large cut-off is that they found a $T_{c}$ dome with $T_{c}$ as large as $0.45 \mathrm{~K}$ even for densities as low as $10^{17} \mathrm{~cm}^{-3}$, in clear disagreement with experiment (see the green curve in their Fig. 2).

In Appendix A.3 the authors of Ref. 1 attempted to justify their procedure by arguing that the vertex correction is small because the coupling is weak. However, they used a full screened interaction, taking the static limit of screening not only for the electrons, but also for the phonons in their eq.A34. We note that according to ref 4 the attraction comes precisely from the frequency 
dependence of the phonon part of the dielectric function $\epsilon_{p h}(\omega)=\epsilon_{\infty}\left(\omega_{L O}^{2}+\omega_{n}^{2}\right) /\left(\omega_{T O}^{2}+\omega_{n}^{2}\right)$ where $\omega_{n}$ is the Matsubara frequency. The attraction comes from the difference in potential with the dielectric function evaluated between the frequencies $\omega_{L O}$ and $\omega_{T O}$. This difference is much larger than the staic limit used in Ref. 1. By using the static lomit, these authors have under-estimated the couplng by orders of magnitude. The correct estimate should be taken at a range of frequencies up to the LO mode and the vertex correction is non-negligible. Thus, there is no reason why only the ladder diagrams can be kept and the results of using the Eliashberg equation up to high cut-off are generically inaccurate.

\section{ACKNOWLEDGMENTS}

We thank Peter Wölfle for pointing out Ref. 11. JR acknowledges work to appear with Zhen Bi and Vladyslav Kozii which is closely related to some of the results presented here. PAL acknowledges the support of DOE under grant no. FG02-03-ER46076.
1 P. Wölfle and A. V. Balatsky, Physical Review B 98, 104505 (2018).

2 The objective of Ref. 1 was to fill in a gap formed in Ref. 15, where it was argued that the paraelectric-ferroelectric quantum critical point in $\mathrm{STO}^{16}$ is the origin of the superconducting dome without specifying the microscopic coupling between the Fermi surface and the soft TO mode.

3 J. Ruhman and P. A. Lee, Physical Review B 94, 224515 (2016), arXiv:1605.01737.

${ }^{4}$ L. V. Gurevich, A. I. Larkin, and Y. A. Firsov, Sov. Phys. Sol. State 4, 131 (1962).

${ }^{5}$ D. E. Khmelnitskii and V. L. Shneerson, Soviet Physics Solid State, USSR 13, 687 (1971).

${ }^{6}$ B. A. Strukov and A. P. Levanyuk, Ferroelectric phenomena in crystals: physical foundations (Springer Science \& Business Media, 2012).
7 O. Kvyatkovskii, Physics of the Solid State 43, 1401 (2001).

8 R. Roussev and A. Millis, Physical Review B 67, 014105 (2003).

9 J. Appel, Phys. Rev. 180, 508 (1969).

10 A. Verma, A. Kajdos, T. Cain, S. Stemmer, and D. Jena, Phys. Rev. Lett. 112, 216601 (2014).

11 J.-J. Zhou, O. Hellman, and M. Bernardi, Phys. Rev. Lett. 121, 226603 (2018).

12 X. Lin, B. Fauqué, and K. Behnia, Science 349, 945 (2015).

13 M. Grabowski and L. J. Sham, Phys. Rev. B 29, 6132 (1984).

14 Y. Takada, Journal of the Physical Society of Japan 49, 1267 (1980).

15 J. M. Edge, Y. Kedem, U. Aschauer, N. A. Spaldin, and A. V. Balatsky, Phys. Rev. Lett. 115, 247002 (2015).

16 S. E. Rowley, L. J. Spalek, R. P. Smith, M. P. M. Dean, M. Itoh, J. F. Scott, G. G. Lonzarich, and S. S. Saxena, Nature Physics 10, 1745 (2014), arXiv:0903.1445. 